\title{
A FINITE ELEMENT METHOD FOR EXTENDED KDV EQUATIONS
}

\author{
ANNA KARCZEWSKA ${ }^{a}$, PiOtR ROZMEJ $^{b, *}$, MACIEJ SZCZECIŃSKI ${ }^{a}$, \\ BARTOSZ BOGUNIEWICZ ${ }^{b}$
}

\author{
${ }^{a}$ Faculty of Mathematics, Computer Science and Econometrics \\ University of Zielona Góra, Szafrana 4a, 65-516 Zielona Góra, Poland \\ email: \{a.karczewska,m.szczecinski\}@wmie.uz.zgora.pl \\ ${ }^{b}$ Institute of Physics, Faculty of Physics and Astronomy \\ University of Zielona Góra, Szafrana 4a, 65-516 Zielona Góra, Poland \\ email:p.rozmej@if.uz.zgora.pl,b.boguniewicz@gmail.com
}

\begin{abstract}
The finite element method (FEM) is applied to obtain numerical solutions to a recently derived nonlinear equation for the shallow water wave problem. A weak formulation and the Petrov-Galerkin method are used. It is shown that the FEM gives a reasonable description of the wave dynamics of soliton waves governed by extended KdV equations. Some new results for several cases of bottom shapes are presented. The numerical scheme presented here is suitable for taking into account stochastic effects, which will be discussed in a subsequent paper.
\end{abstract}

Keywords: shallow water wave problem, nonlinear equations, second order KdV equations, finite element method, PetrovGalerkin method.

\section{Introduction}

The Korteveg-de Vries equation appears as a model for the propagation of weakly nonlinear dispersive waves in several fields. Among them, there are gravity driven waves on a surface of an incompressible irrotational inviscid fluid (Whitham, 1974; Infeld and Rowlands, 2000; Remoissenet, 1999; Drazin and Johnson, 1989; Marchant and Smyth, 1990; Dingemans, 1997), ion acoustic waves in plasma (Infeld and Rowlands, 2000), impulse propagation in electric circuits (Remoissenet, 1999), and so on. In the shallow water wave problem the $\mathrm{KdV}$ equation corresponds to the case when the bottom is even. There have been many attempts to study nonlinear waves in the case of an uneven bottom because of its significance, for instance, in phenomena such as tsunamis. Among the first papers dealing with a slowly varying bottom are those of Mei and Le Méhauté (1966) as well as Grimshaw (1970). When taking an appropriate average of vertical variables, one arrives at Green-Nagdi type equations (Green and Naghdi, 1976; Nadiga et al., 1996; Kim et al., 2001). Van Groesen and Pudyaprasetya $(1993 ; 1996)$ studied uni-directional waves over a slowly

*Corresponding author varying bottom within the Hamilton approach, obtaining a forced $\mathrm{KdV}$ type equation. An extensive study of wave propagation over an uneven bottom conducted before 2000 is summarized in Dingemans's monograph (Dingemans, 1997). The papers by Nakoulima et al. (2005), Grimshaw et al. (2008) and Pelinovsky et al. (2010) are examples of approaches that combine linear and nonlinear theories. The Gardner equation and the forced $\mathrm{KdV}$ equation were also extensively investigated in this context (see, e.g., Grimshaw and Smyth, 1986; Smyth, 1987; Kamchatnov et al., 2012).

In our previous papers (Karczewska et al., 2014b; 2014a) we derived a new KdV type equation containing terms which come directly from an uneven bottom. These terms, however, appear naturally only if Euler equations for the fluid motion are considered up to the second order in small parameters, whereas the $\mathrm{KdV}$ equation is obtained in a first order approximation. There are no analytic solutions for the above equation. We also presented several cases of numerical simulations for that equation obtained using the finite difference method (FDM) with periodic boundary conditions (Karczewska et $a l ., 2014 b ; 2014 a)$. 
It was demonstrated by Debussche and Printems (1999) that the finite element method (FEM) describes properly the dynamics of the $\mathrm{KdV}$ equation (6), which is the equation in a moving frame of reference.

The first aim of this paper is to construct an effective FEM method for solving higher order $\mathrm{KdV}$ equations, both with an even and an uneven bottom. The second goal is to compare the results obtained in this numerical scheme with some of the results obtained earlier using the finite difference method (Karczewska et al., 2014b; 2014a).

The paper is organized as follows. In Section 2 we review the $\mathrm{KdV}$ equation (4), the extended $\mathrm{KdV}$ equation (3) and the $\mathrm{KdV}$ type equation containing direct terms from bottom variation (1), all expressed in scaled dimensionless variables. In Section 3 the construction of the numerical method for solving these equations within the FEM is described. Coupled sets of nonlinear equations for coefficients of expansion of solutions to these equations in a basis of piecewise linear functions are obtained. In Section 4 several examples of numerical simulations are presented.

\section{Preliminaries}

Extended KdV type equations, derived by some of the authors (Karczewska et al., 2014b; 2014a), second order in small parameters, have the following form (written in scaled dimensionless coordinates, in a fixed coordinate system), for the case with an uneven bottom:

$$
\begin{aligned}
\eta_{t} & +\eta_{x}+\alpha \frac{3}{2} \eta \eta_{x}+\beta \frac{1}{6} \eta_{3 x} \\
& -\frac{3}{8} \alpha^{2} \eta^{2} \eta_{x}+\alpha \beta\left(\frac{23}{24} \eta_{x} \eta_{2 x}+\frac{5}{12} \eta \eta_{3 x}\right)+\frac{19}{360} \beta^{2} \eta_{5 x} \\
& +\beta \delta\left(-\frac{1}{2 \beta}(h \eta)_{x}+\frac{1}{4}\left(h_{2 x} \eta\right)_{x}-\frac{1}{4}\left(h \eta_{2 x}\right)_{x}\right)=0 .
\end{aligned}
$$

Details of the derivation of the second order equation (1) from the set of Euler equations with appropriate boundary conditions can be found in the works of Karczewska et al. (2014b; 2014a). In (1), $\eta(x, t)$ stands for a wave profile and $h=h(x)$ denotes a bottom profile. Subscripts are used for notation of partial derivatives, that is, for instance, $\eta_{2 x} \equiv \partial^{2} \eta / \partial x^{2}$, and so on. Small parameters $\alpha, \beta, \delta$ are defined by ratios of the amplitude of the wave profile $a$, the depth of undisturbed water $h_{0}$, average wavelength $l$ and the amplitude of the bottom changes $a_{h}$ :

$$
\alpha=\frac{a}{h_{0}}, \quad \beta=\left(\frac{h}{l}\right)^{2}, \quad \delta=\frac{a_{h}}{h_{0}} .
$$

For details of the transformation of the original dimensional variables to the nondimensional, scaled ones used here, see, e.g., the works of Karczewska et al. (2014b; 2014a) or Burde and Sergyeyev (2013).
It should be emphasized that in Eqn. (11) all the three terms originating from an uneven bottom are second order in small parameters. These terms appear from the boundary condition at the bottom, which is already in the second order with the coefficient $\beta \delta$ (see Karczewska et al., 2014a, Eqn. (5); 2014b, Eqn. (10)). Then in the final second order equation (1) we write them in the form $\beta \delta(\cdot)$ in order to emphasize that they all come from the second order perturbation approach. For details we refer to the above-mentioned papers.

In the case of an even bottom $(\delta=0)$, Eqn. (1) is reduced to the second order $\mathrm{KdV}$ type equation,

$$
\begin{aligned}
\eta_{t} & +\eta_{x}+\alpha \frac{3}{2} \eta \eta_{x}+\beta \frac{1}{6} \eta_{3 x}-\frac{3}{8} \alpha^{2} \eta^{2} \eta_{x} \\
& +\alpha \beta\left(\frac{23}{24} \eta_{x} \eta_{2 x}+\frac{5}{12} \eta \eta_{3 x}\right)+\frac{19}{360} \beta^{2} \eta_{5 x}=0
\end{aligned}
$$

and, when $\beta=\alpha$, it becomes identical to Eqn. (21) of Burde and Sergyeyev (2013). Equation (3) was obtained earlier by Marchant and Smyth (1990) and is called the extended $K d V$ equation.

Limitation to the first order approximation in small parameters gives $\mathrm{KdV}$ in a fixed system of coordinates

$$
\eta_{t}+\eta_{x}+\alpha \frac{3}{2} \eta \eta_{x}+\beta \frac{1}{6} \eta_{3 x}=0 .
$$

The standard, mathematical form of the $\mathrm{KdV}$ equation is obtained from (4) by transformation to a moving reference frame. Substituting

$$
\bar{x}=\sqrt{\frac{3}{2}}(x-t), \quad \bar{t}=\frac{1}{4} \sqrt{\frac{3}{2}} \alpha t, \quad u=\eta,
$$

one obtains from (3) the equation

$$
u_{\bar{t}}+6 u u_{\bar{x}}+\frac{\beta}{\alpha} u_{3 \bar{x}}=0,
$$

or finally, when $\beta=\alpha$,

$$
u_{\bar{t}}+6 u u_{\bar{x}}+u_{3 \bar{x}}=0 .
$$

In this paper we attempt to solve numerically Eqn. (1) for several cases of bottom topography and different initial conditions. In several aspects we follow the method applied by Debussche and Printems (1999). However, the method is extended to higher order $\mathrm{KdV}$ type equations with a plain bottom (3) and with bottom fluctuations (1). For both the cases we work in a fixed reference system, necessary for a bottom profile depending on the position.

\section{Numerical method}

The emergence of soliton solutions to the KdV equation was observed in numerics fifty years ago by Zabusky and Kruskal (1965). Several numerical methods used 
for solving the $\mathrm{KdV}$ equation are discussed by Taha and Ablowitz (1984). Among them there are the finite difference explicit method (Zabusky and Kruskal, 1965), the finite difference implicit method (Goda, 1975) and several versions of the pseudospectral method (Fornberg and Whitham, 1978). It is also worth mentioning papers using the FEM and Galerkin methods (Bona et al., 2013; Cui and Ma, 2007). Most numerical applications use periodic boundary conditions, but there also exist works that apply Dirichlet boundary conditions on a finite interval (Skogstad and Kalisch, 2009; Yi et al., 2013; Yuan et al., 2008).

The authors attempt to construct a method which will be applicable not only for numerical simulation of an evolution of nonlinear waves governed by Eqns. (1) or (3), but also for their stochastic versions. Such stochastic equations will be studied in the next paper. Since stochastic noise is irregular, solutions are not necessarily smooth, neither in time nor space. A finite element method (FEM) seems to be suitable for such a case.

3.1. Time discretization. We have adapted the Crank-Nicholson scheme for time evolution, beginning with the $\mathrm{KdV}$ equation (4) in a fixed coordinate system. Note that $\eta \eta_{x}=\frac{1}{2}\left(\eta^{2}\right)_{x}$. Write also $v:=\eta_{x}$ and $w:=v_{x}$. Let us choose, time step $\tau$. Then the KdV equation (4) in the Crank-Nicholson scheme can be written as a set of coupled first order differential equations,

$$
\begin{aligned}
\eta^{n+1}-\eta^{n}+\tau\left(\frac{\partial}{\partial x} \eta^{n+\frac{1}{2}}\right. & \\
\left.+\frac{3 \alpha}{4} \frac{\partial}{\partial x}\left(\eta^{n+\frac{1}{2}}\right)^{2}+\frac{\beta}{6} w^{n+\frac{1}{2}}\right) & =0 \\
\frac{\partial}{\partial x} \eta^{n+\frac{1}{2}} & =v^{n+\frac{1}{2}} \\
\frac{\partial}{\partial x} v^{n+\frac{1}{2}} & =w^{n+\frac{1}{2}}
\end{aligned}
$$

where

$$
\begin{aligned}
\eta^{n+\frac{1}{2}} & =\frac{1}{2}\left(\eta^{n+1}+\eta^{n}\right), \\
v^{n+\frac{1}{2}} & =\frac{1}{2}\left(v^{n+1}+v^{n}\right), \\
w^{n+\frac{1}{2}} & =\frac{1}{2}\left(w^{n+1}+w^{n}\right) .
\end{aligned}
$$

For the second order equations (1) or (3) we need to introduce two new auxiliary variables: $p:=w_{x}$ and $q:=p_{x}$. Note that $\eta^{2} \eta_{x}=\frac{1}{3}\left(\eta^{3}\right)_{x}, \eta_{x} \eta_{2 x}=\frac{1}{2}\left(\eta_{x}^{2}\right)_{x}=$ $\frac{1}{2}\left(v^{2}\right)_{x}$. Moreover, $\eta_{5 x}=q=p_{x}$ and

$$
\frac{23}{24} \eta_{x} \eta_{2 x}+\frac{5}{12} \eta \eta_{3 x}=\frac{13}{48}\left(v^{2}\right)_{x}+\frac{5}{12}(\eta w)_{x} .
$$

This setting allows us to write the Crank-Nicholson scheme for (3) as the following set of first order equations:

$$
\begin{aligned}
& \eta^{n+1}-\eta^{n} \\
&+\tau \frac{\partial}{\partial x}\left[\eta^{n+\frac{1}{2}}+\frac{3 \alpha}{4}\left(\eta^{n+\frac{1}{2}}\right)^{2}+\frac{\beta}{6} w^{n+\frac{1}{2}}\right. \\
&-\frac{1}{8} \alpha^{2}\left(\eta^{n+\frac{1}{2}}\right)^{3}+\alpha \beta\left(\frac{13}{48}\left(v^{n+\frac{1}{2}}\right)^{2}\right. \\
&\left.\left.+\frac{5}{12}\left(\eta^{n+\frac{1}{2}} w^{n+\frac{1}{2}}\right)\right)+\frac{19}{360} \beta^{2}\left(q^{n+\frac{1}{2}}\right)\right]=0, \\
& \frac{\partial}{\partial x} \eta^{n+\frac{1}{2}}-v^{n+\frac{1}{2}}=0, \\
& \frac{\partial}{\partial x} v^{n+\frac{1}{2}}-w^{n+\frac{1}{2}}=0, \\
& \frac{\partial}{\partial x} w^{n+\frac{1}{2}}-p^{n+\frac{1}{2}}=0, \\
& \frac{\partial}{\partial x} p^{n+\frac{1}{2}}-q^{n+\frac{1}{2}}=0,
\end{aligned}
$$

where

$$
\begin{aligned}
& p^{n+\frac{1}{2}}=\frac{1}{2}\left(p^{n+1}+p^{n}\right), \\
& q^{n+\frac{1}{2}}=\frac{1}{2}\left(q^{n+1}+q^{n}\right) .
\end{aligned}
$$

For the second order $\mathrm{KdV}$ type equation with an uneven bottom (1) the first equation in the set (9) has to be supplemented by terms originating from bottom variations, yielding

$$
\begin{gathered}
\eta^{n+1}-\eta^{n} \\
+\tau \frac{\partial}{\partial x}\left[\eta^{n+\frac{1}{2}}+\frac{3 \alpha}{4}\left(\eta^{n+\frac{1}{2}}\right)^{2}+\frac{\beta}{6} w^{n+\frac{1}{2}}\right. \\
-\frac{1}{8} \alpha^{2}\left(\eta^{n+\frac{1}{2}}\right)^{3}+\alpha \beta\left(\frac{13}{48}\left(v^{n+\frac{1}{2}}\right)^{2}\right. \\
\left.+\frac{5}{12}\left(\eta^{n+\frac{1}{2}} w^{n+\frac{1}{2}}\right)\right)+\frac{19}{360} \beta^{2}\left(q^{n+\frac{1}{2}}\right) \\
\frac{1}{4} \beta \delta\left(-\frac{2}{\beta}\left(h^{n+\frac{1}{2}} \eta^{n+\frac{1}{2}}\right)\right. \\
\left.\left.+\eta^{n+\frac{1}{2}} g^{n+\frac{1}{2}}+h^{n+\frac{1}{2}} w^{n+\frac{1}{2}}\right)\right]=0,
\end{gathered}
$$

where $g:=h_{x x}$.

Below we focus on the second order equations (3) and (9), pointing out contributions from the bottom variation later.

3.2. Space discretization. Following the arguments given by Debussche and Printems (1999) we apply the Petrov-Galerkin discretization and the finite element method. We use piecewise linear shape functions and piecewise constant test functions. We consider the wave motion on the interval $x \in[0, L]$ with periodic boundary conditions. Given $N \in \mathbb{N}$, we use a mesh $M_{\chi}$ of points $x_{j}=j \chi, j=0,1, \ldots, N$, where $\chi=L / N$. Let $V_{\chi}^{1}$, 
which is a space of piecewise linear functions $\varphi_{j}(x)$, such that $\varphi_{j}(0)=\varphi_{j}(L)$, defined as

$$
\varphi_{j}(x)= \begin{cases}\frac{1}{\chi}\left(x-x_{j-1}\right) & \text { if } x \in\left[x_{j-1}, x_{j}\right], \\ \frac{1}{\chi}\left(x_{j+1}-x\right) & \text { if } x \in\left[x_{j}, x_{j+1}\right], \\ 0 & \text { otherwise. }\end{cases}
$$

As test functions we have chosen the space of piecewise constant functions $\psi_{j}(x) \in V_{\chi}^{0}$, where

$$
\psi_{j}(x)= \begin{cases}1 & \text { if } x \in\left[x_{j}, x_{j+1}\right) \\ 0 & \text { otherwise. }\end{cases}
$$

An approximate solution and its derivatives may be written as an expansion in the basis (12),

$$
\begin{aligned}
& \eta_{\chi}^{n}(x)=\sum_{j=1}^{N} a_{j}^{n} \varphi_{j}(x), \\
& v_{\chi}^{n}(x)=\sum_{j=1}^{N} b_{j}^{n} \varphi_{j}(x), \\
& w_{\chi}^{n}(x)=\sum_{j=1}^{N} c_{j}^{n} \varphi_{j}(x), \\
& p_{\chi}^{n}(x)=\sum_{j=1}^{N} d_{j}^{n} \varphi_{j}(x), \\
& q_{\chi}^{n}(x)=\sum_{j=1}^{N} e_{j}^{n} \varphi_{j}(x),
\end{aligned}
$$

where $a_{j}^{n}, b_{j}^{n}, c_{j}^{n}, d_{j}^{n}, e_{j}^{n}$ are expansion coefficients. Therefore, in a weak formulation we can write (8) as

$$
\begin{aligned}
\left(\eta_{\chi}^{n+1}-\eta_{\chi}^{n}, \psi_{i}\right)+ & \left\{\left(\partial_{x} \eta_{\chi}^{n+\frac{1}{2}}, \psi_{i}\right)\right. \\
+\frac{3 \alpha}{4}\left(\partial_{x}\left(\eta_{\chi}^{n+\frac{1}{2}}\right)^{2}, \psi_{i}\right)+\frac{\beta}{6}\left(\partial_{x} w_{\chi}^{n+\frac{1}{2}}, \psi_{i}\right) & -\frac{1}{8} \alpha^{2}\left(\partial_{x}\left(\eta_{\chi}^{n+\frac{1}{2}}\right)^{3}, \psi_{i}\right) \\
+ & \alpha \beta\left[\frac{13}{48}\left(\partial_{x}\left(v_{\chi}^{n+\frac{1}{2}}\right)^{2}, \psi_{i}\right)\right. \\
+ & \left.\frac{5}{12}\left(\partial_{x}\left(\eta_{\chi}^{n+\frac{1}{2}} w_{\chi}^{n+\frac{1}{2}}\right), \psi_{i}\right)\right] \\
+ & \left.\frac{19}{360} \beta^{2}\left(\partial_{x}\left(q_{\chi}^{n+\frac{1}{2}}\right), \psi_{i}\right)\right\}=0, \\
& \left(\partial_{x} \eta_{\chi}^{n+\frac{1}{2}}, \psi_{i}\right)-\left(v_{\chi}^{n+\frac{1}{2}}, \psi_{i}\right)=0, \\
& \left(\partial_{x} v_{\chi}^{n+\frac{1}{2}}, \psi_{i}\right)-\left(w_{\chi}^{n+\frac{1}{2}}, \psi_{i}\right)=0, \\
& \left(\partial_{x} w_{\chi}^{n+\frac{1}{2}}, \psi_{i}\right)-\left(p_{\chi}^{n+\frac{1}{2}}, \psi_{i}\right)=0, \\
& \left(\partial_{x} p_{\chi}^{n+\frac{1}{2}}, \psi_{i}\right)-\left(q_{\chi}^{n+\frac{1}{2}}, \psi_{i}\right)=0,
\end{aligned}
$$

for any $i=1, \ldots, N$, where $\partial_{x}$ is understood as $\partial / \partial x$. In (15) and below, scalar products are defined by the appropriate integrals:

$$
(f, g):=\int_{0}^{L} f(x) g(x) \mathrm{d} x .
$$

In the case of Eqn. (1), the first equation of the set (15) has to be supplemented inside the bracket \{\} by the terms

$$
\begin{aligned}
+\frac{1}{4} \beta \delta\left(\partial_{x}[\right. & -\frac{2}{\beta}\left(h^{n+\frac{1}{2}} \eta_{\chi}^{n+\frac{1}{2}}\right) \\
& \left.\left.+\eta_{\chi}^{n+\frac{1}{2}} g^{n+\frac{1}{2}}+h^{n+\frac{1}{2}} w_{\chi}^{n+\frac{1}{2}}\right], \psi_{i}\right) .
\end{aligned}
$$

Insertion of (14) into 15 yields a system of coupled linear equations for coefficients $a_{j}^{n}, b_{j}^{n}, c_{j}^{n}, d_{j}^{n}, e_{j}^{n}$. The solution to this system supplies an approximate solution to (3) given at the mesh points $x_{j}$.

3.2.1. KdV equation. In order to demonstrate the construction of the matrices involved, at this point we limit our discussion to the first order equation (4). This means that in (15) we drop temporarily second order terms, that is, those with $\alpha^{2}, \alpha \beta, \beta^{2}$. Equations with $p$ and $q$ do not apply because $\eta_{4 x}$ and $\eta_{5 x}$ do not appear in (4). This leads to the equations

$$
\begin{gathered}
\sum_{j=1}^{N}\left(a_{j}^{n+1}-a_{j}^{n}\right)\left(\varphi_{j}, \psi_{i}\right)+\tau \frac{1}{2} \sum_{j=1}^{N}\left(b_{j}^{n+1}+b_{j}^{n}\right)\left(\varphi_{j}, \psi_{i}\right) \\
+\tau \alpha \frac{3}{16} \sum_{j=1}^{N} \sum_{k=1}^{N}\left(a_{j}^{n+1}+a_{j}^{n}\right)\left(a_{k}^{n+1}+a_{k}^{n}\right) \\
\times\left(\varphi_{j}^{\prime} \varphi_{k}+\varphi_{j} \varphi_{k}^{\prime}, \psi_{i}\right) \\
+\tau \beta \frac{1}{12} \sum_{j=1}^{N}\left(c_{j}^{n+1}+c_{j}^{n}\right)\left(\varphi_{j}, \psi_{i}\right)=0, \\
\sum_{j=1}^{N}\left[\left(a_{j}^{n+1}+a_{j}^{n}\right)\left(\varphi_{j}^{\prime}, \psi_{i}\right)-\left(b_{j}^{n+1}+b_{j}^{n}\right)\left(\varphi_{j}, \psi_{i}\right)\right]=0, \\
\sum_{j=1}^{N}\left[\left(b_{j}^{n+1}+b_{j}^{n}\right)\left(\varphi_{j}^{\prime}, \psi_{i}\right)-\left(c_{j}^{n+1}+c_{j}^{n}\right)\left(\varphi_{j}, \psi_{i}\right)\right]=0 .
\end{gathered}
$$

Define

$$
\begin{array}{ll}
C_{i j}^{(1)}:=\left(\varphi_{j}, \psi_{i}\right), & C_{i j}^{(2)}:=\left(\varphi_{j}^{\prime}, \psi_{i}\right), \\
C_{i j k}^{(3)} & :=\left(\varphi_{j}^{\prime} \varphi_{k}+\varphi_{j} \varphi_{k}^{\prime}, \psi_{i}\right),
\end{array}
$$

where $\varphi_{j}^{\prime}=\frac{\mathrm{d} \varphi}{\mathrm{d} x}\left(x_{j}\right)$. Simple integration shows that

$$
C_{i j}^{(1)}=\left\{\begin{aligned}
\frac{1}{2} \chi & \text { if } i=j \vee i=j-1 \\
0 & \text { otherwise }
\end{aligned}\right.
$$




$$
C_{i j}^{(2)}=\left\{\begin{aligned}
-1 & \text { if } \quad i=j, \\
1 & \text { if } i=j-1, \\
0 & \text { otherwise. }
\end{aligned}\right.
$$

Similarly, one obtains

$$
C_{i j k}^{(3)}=C_{i j}^{(2)} \delta_{j k} .
$$

The property 211 reduces the double sum in the term with $\tau \alpha \frac{3}{16}$ to the single one of the square of $\left(a_{j}^{n+1}+a_{j}^{n}\right)$. Insertion of 19-121) into (17) gives

$$
\begin{aligned}
\sum_{j=1}^{N}\left[\left(a_{j}^{n+1}-a_{j}^{n}\right) C_{i j}^{(1)}+\tau\left(\frac{1}{2}\left(b_{j}^{n+1}+b_{j}^{n}\right) C_{i j}^{(1)}\right.\right. & \\
\left.\left.+\alpha \frac{3}{16}\left(a_{j}^{n+1}+a_{j}^{n}\right)^{2} C_{i j}^{(2)}+\beta \frac{1}{12}\left(c_{j}^{n+1}+c_{j}^{n}\right) C_{i j}^{(2)}\right)\right] & =0, \\
\sum_{j=1}^{N}\left[\left(a_{j}^{n+1}+a_{j}^{n}\right) C_{i j}^{(2)}-\left(b_{j}^{n+1}+b_{j}^{n}\right) C_{i j}^{(1)}\right] & =0, \\
\sum_{j=1}^{N}\left[\left(b_{j}^{n+1}+b_{j}^{n}\right) C_{i j}^{(2)}-\left(c_{j}^{n+1}+c_{j}^{n}\right) C_{i j}^{(1)}\right] & =0 .
\end{aligned}
$$

Define the $3 N$-dimensional vector of expansion coefficients

$$
X^{n}=\left(\begin{array}{c}
A^{n} \\
B^{n} \\
C^{n}
\end{array}\right),
$$

where

$$
A^{n}=\left(\begin{array}{c}
a_{1}^{n} \\
a_{2}^{n} \\
\vdots \\
a_{N}^{n}
\end{array}\right), \quad B^{n}=\left(\begin{array}{c}
b_{1}^{n} \\
b_{2}^{n} \\
\vdots \\
b_{N}^{n}
\end{array}\right), \quad C^{n}=\left(\begin{array}{c}
c_{1}^{n} \\
c_{2}^{n} \\
\vdots \\
c_{N}^{n}
\end{array}\right) .
$$

In (22), $A^{n+1}, B^{n+1}, C^{n+1}$ represent the unknown coefficients and $A^{n}, B^{n}, C^{n}$ the known ones. Note that the system (22) is nonlinear. The single nonlinear term is quadratic in unknown coefficients. For the second order equations (3) and (1) there are more nonlinear terms.

In an abbreviated form, the set (22) can be written as

$$
F_{i}\left(X^{n+1}, X^{n}\right)=0, \quad i=1,2, \ldots, 3 N .
$$

Since this equation is nonlinear, we can use the Newton method for each time step. That is, we find $X^{n+1}$ by iterating the equation

$$
\left(X^{n+1}\right)_{m+1}=\left(X^{n+1}\right)_{m}+J^{-1}\left(X^{n+1}\right)_{m}=0,
$$

where $J^{-1}$ is the inverse of the Jacobian of $F\left(X^{n+1}, X^{n}\right)$ (25). Choosing $\left(X^{n+1}\right)_{0}=X^{n}$, we obtain the approximate solution to (25), $\left(X^{n+1}\right)_{m}$ in $m=3-5$ iterations with very good precision. The Jacobian itself is a particular $(3 N \times 3 N)$ sparse matrix with the following block structure:

$$
J=\left(\begin{array}{ccc}
\left(A_{a}\right) & \left(A_{b}\right) & \left(A_{c}\right) \\
(C 2) & -(C 1) & (0) \\
(0) & (C 2) & -(C 1)
\end{array}\right),
$$

where each block $(\cdot)$ is a two-diagonal sparse $(N \times N)$ matrix. The matrix $A_{a}$ is given by

$$
A_{a}=\left(\begin{array}{ccccccc}
a_{1}^{1} & 0 & 0 & \cdots & 0 & a_{N-1}^{1} & a_{N}^{1} \\
a_{1}^{2} & a_{2}^{2} & 0 & \cdots & 0 & 0 & a_{N}^{2} \\
0 & a_{2}^{3} & a_{3}^{3} & 0 & \cdots & 0 & 0 \\
\vdots & \vdots & \vdots & \ddots & \vdots & \vdots & \vdots \\
0 & 0 & \cdots & a_{N-4}^{N-3} & a_{N-3}^{N-3} & 0 & 0 \\
0 & 0 & \cdots & 0 & a_{N-3}^{n-2} & a_{N-2}^{N-2} & 0 \\
a_{1}^{N} & 0 & \cdots & 0 & 0 & a_{N}^{N-1} & a_{N}^{n}
\end{array}\right) .
$$

In (28) the nonzero elements of $A_{a}$ are expressed by

$$
a_{j}^{i}=\frac{\partial F_{i}}{\partial a_{j}^{n+1}},
$$

where $F$ is given by (25). The elements in the upper right and lower left corners result from periodic boundary conditions. Matrices $A_{b}$ and $A_{c}$ have the same structure as $A_{a}$, with only elements $a_{j}^{i}$ having to be replaced by $b_{j}^{i}=\frac{\partial F_{i}}{\partial b_{j}^{n+1}}$ and $c_{j}^{i}=\frac{\partial F_{i}}{\partial c_{j}^{n+1}}$, respectively.

Matrices $C 1$ and $C 2$ are constant. They are defined as

$$
C k=\left(\begin{array}{ccccc}
C_{11}^{(k)} & 0 & \cdots & C_{11}^{(k)} & C_{1 N}^{(k)} \\
C_{21}^{(k)} & C_{22}^{(k)} & \cdots & 0 & C_{2 N}^{(k)} \\
\vdots & \vdots & \ddots & \vdots & \vdots \\
0 & 0 & \cdots & C_{N-1 N-1}^{(k)} & 0 \\
C_{N 1}^{(k)} & 0 & \cdots & C_{N-1 N}^{(k)} & C_{N N}^{(k)}
\end{array}\right)
$$

where $k=1,2$.

3.2.2. Extended KdV equation (3). For the second order equation (3) there are more nonlinear terms. These are terms with $\alpha^{2}$ and $\alpha \beta$. According to the Petrov-Galerkin scheme, we get for the term with $\alpha^{2}$

$$
\begin{aligned}
& \partial_{x}\left(\eta^{n+\frac{1}{2}}\right)^{3}=\frac{1}{8}\left(\partial_{x} \sum_{j=1}^{N}\left(a_{j}^{n+1}+a_{j}^{n}\right) \varphi_{j}\right)^{3} \\
& =\frac{1}{8} \partial_{x} \sum_{j=1}^{N} \sum_{k=1}^{N} \sum_{l=1}^{N}\left[a_{j}^{n+1}+a_{j}^{n}\right]\left[a_{k}^{n+1}+a_{k}^{n}\right]\left[a_{l}^{n+1}+a_{l}^{n}\right] \\
& \quad \times \varphi_{j} \varphi_{k} \varphi_{l} \\
& =\frac{1}{8} \sum_{j=1}^{N} \sum_{k=1}^{N} \sum_{l=1}^{N}\left[a_{j}^{n+1}+a_{j}^{n}\right]\left[a_{k}^{n+1}+a_{k}^{n}\right]\left[a_{l}^{n+1}+a_{l}^{n}\right] \\
& \quad \times\left(\varphi_{j}^{\prime} \varphi_{k} \varphi_{l}+\varphi_{j} \varphi_{k}^{\prime} \varphi_{l}+\varphi_{j} \varphi_{k} \varphi_{l}^{\prime}\right) .
\end{aligned}
$$


Write

$$
C_{i j k l}^{(4)}:=\left(\left[\varphi_{j}^{\prime} \varphi_{k} \varphi_{l}+\varphi_{j} \varphi_{k}^{\prime} \varphi_{l}+\varphi_{j} \varphi_{k} \varphi_{l}^{\prime}\right], \psi_{i}\right) .
$$

As with $C_{i j k}^{(3)}$ in (21), the following property holds:

$$
C_{i j k l}^{(4)}=C_{i j}^{2} \delta_{j k} \delta_{k l}
$$

In a similar way, for terms with $\alpha \beta$ we obtain

$$
\begin{aligned}
& \partial_{x}\left(v^{n+\frac{1}{2}}\right)^{2} \\
& =\frac{1}{4} \partial_{x}\left(\sum_{j=1}^{N}\left(b_{j}^{n+1}+b_{j}^{n}\right) \varphi_{j} \sum_{k=1}^{N}\left(b_{k}^{n+1}+b_{k}^{n}\right) \varphi_{k}\right) \\
& =\frac{1}{4} \sum_{j=1}^{N} \sum_{k=1}^{N}\left[b_{j}^{n+1}+a_{j}^{n}\right]\left[b_{k}^{n+1}+b_{k}^{n}\right]\left(\varphi_{j}^{\prime} \varphi_{k}+\varphi_{j} \varphi_{k}^{\prime}\right)
\end{aligned}
$$

and

$$
\begin{aligned}
& \partial_{x}\left(\eta^{n+\frac{1}{2}} w^{n+\frac{1}{2}}\right) \\
& =\frac{1}{4} \partial_{x}\left(\sum_{j=1}^{N}\left(a_{j}^{n+1}+a_{j}^{n}\right) \varphi_{j} \sum_{k=1}^{N}\left(a_{k}^{n+1}+a_{k}^{n}\right) \varphi_{k}\right) \\
& =\frac{1}{4} \sum_{j=1}^{N} \sum_{k=1}^{N}\left[a_{j}^{n+1}+a_{j}^{n}\right]\left[b_{k}^{n+1}+b_{k}^{n}\right]\left(\varphi_{j}^{\prime} \varphi_{k}+\varphi_{j} \varphi_{k}^{\prime}\right) .
\end{aligned}
$$

The scalar products appearing in the terms proportional to $\alpha^{2}$ and $\alpha \beta$ are already defined: $\left(\left(\varphi_{j}^{\prime} \varphi_{k}+\varphi_{j} \varphi_{k}^{\prime}\right), \psi_{i}\right)=$ $C_{i j k}^{(3)}$.

Due to the properties (33) and (21), triple and double sums reduce to single ones. With these settings the second order $\mathrm{KdV}$ equation (15) gives the following system of equations:

$$
\begin{gathered}
\sum_{j=1}^{N}\left\{\left(a_{j}^{n+1}-a_{j}^{n}\right) C_{i j}^{(1)}+\tau\left[\frac{1}{2}\left(b_{j}^{n+1}+b_{j}^{n}\right) C_{i j}^{(1)}\right.\right. \\
+\left(\alpha \frac{3}{16}\left(a_{j}^{n+1}+a_{j}^{n}\right)^{2}+\beta \frac{1}{12}\left(c_{j}^{n+1}+c_{j}^{n}\right)\right. \\
-\alpha^{2} \frac{1}{64}\left(a_{j}^{n+1}+a_{j}^{n}\right)^{3}+\alpha \beta \frac{13}{192}\left(b_{j}^{n+1}+b_{j}^{n}\right)^{2} \\
+\alpha \beta \frac{5}{96}\left(a_{j}^{n+1}+a_{j}^{n}\right)\left(c_{j}^{n+1}+c_{j}^{n}\right) \\
\left.\left.\left.+\beta^{2} \frac{19}{720}\left(e_{j}^{n+1}+e_{j}^{n}\right)\right) C_{i j}^{(2)}\right]\right\}=0,
\end{gathered}
$$

$$
\begin{aligned}
& \sum_{j=1}^{N}\left[\left(a_{j}^{n+1}+a_{j}^{n}\right) C_{i j}^{(2)}-\left(b_{j}^{n+1}+b_{j}^{n}\right) C_{i j}^{(1)}\right]=0, \\
& \sum_{j=1}^{N}\left[\left(b_{j}^{n+1}+b_{j}^{n}\right) C_{i j}^{(2)}-\left(c_{j}^{n+1}+c_{j}^{n}\right) C_{i j}^{(1)}\right]=0, \\
& \sum_{j=1}^{N}\left[\left(c_{j}^{n+1}+c_{j}^{n}\right) C_{i j}^{(2)}-\left(d_{j}^{n+1}+d_{j}^{n}\right) C_{i j}^{(1)}\right]=0, \\
& \sum_{j=1}^{N}\left[\left(b_{j}^{n+1}+b_{j}^{n}\right) C_{i j}^{(2)}-\left(e_{j}^{n+1}+e_{j}^{n}\right) C_{i j}^{(1)}\right]=0,
\end{aligned}
$$

where $i=1,2, \ldots, N$.

In this case the vector of expansion coefficients $X^{n}$ is $5 N$-dimensional,

$$
X^{n}=\left(\begin{array}{c}
A^{n} \\
B^{n} \\
C^{n} \\
D^{n} \\
E^{n}
\end{array}\right)
$$

where $A^{n}, B^{n}$ and $C^{n}$ are already defined in (24) and

$$
D^{n}=\left(\begin{array}{c}
d_{1}^{n} \\
d_{2}^{n} \\
\vdots \\
d_{N}^{n}
\end{array}\right), \quad E^{n}=\left(\begin{array}{c}
e_{1}^{n} \\
e_{2}^{n} \\
\vdots \\
e_{N}^{n}
\end{array}\right) .
$$

The Jacobian now becomes $(5 N \times 5 N)$-dimensional. Its structure, however, is similar to 27, that is,

$$
J=\left(\begin{array}{ccccc}
\left(A_{a}\right) & \left(A_{b}\right) & \left(A_{c}\right) & (0) & \left(A_{e}\right) \\
(C 2) & -(C 1) & (0) & (0) & (0) \\
(0) & (C 2) & -(C 1) & (0) & (0) \\
(0) & (0) & (C 2) & -(C 1) & (0) \\
(0) & (0) & (0) & (C 2) & -(C 1)
\end{array}\right)
$$

where the matrices $\left(A_{a}\right),\left(A_{b}\right),\left(A_{c}\right)$ are defined as previously and

$$
\left(A_{e}\right)_{i j}=\frac{\partial F_{i}}{\partial e_{j}^{n+1}} .
$$

Now $F_{i}$ represents the set 36 which contains four nonlinear terms.

\subsubsection{Extended KdV equation with an uneven bot-} tom. For the extended $\mathrm{KdV}$ with non-flat bottom we have to include into (36) three additional terms contained in the last line of the formula (1). Expanding the bottom function $h(x)$ and its second derivative $h_{2 x}(x)$ in the basis $\left\{\varphi_{j}(x)\right\}$,

$$
h(x)=\sum_{j=1}^{N} H 0_{j} \varphi_{j}(x), \quad h_{2 x}(x)=\sum_{j=1}^{N} H 2_{j} \varphi_{j}(x),
$$


we can write the terms mentioned above in the following form:

$$
\begin{aligned}
& \partial_{x}\left(h \eta^{n+\frac{1}{2}}\right) \\
& =\frac{1}{2} \sum_{j=1}^{N} \sum_{k=1}^{N} H 0_{j}\left(a_{k}^{n+1}+a_{k}^{n}\right)\left(\varphi_{j}^{\prime} \varphi_{k}+\varphi_{j} \varphi_{k}^{\prime}\right), \\
& \partial_{x}\left(h 2 x \eta^{n+\frac{1}{2}}\right) \\
& =\frac{1}{2} \sum_{j=1}^{N} \sum_{k=1}^{N} H 2_{j}\left(a_{k}^{n+1}+a_{k}^{n}\right)\left(\varphi_{j}^{\prime} \varphi_{k}+\varphi_{j} \varphi_{k}^{\prime}\right) . \\
& \partial_{x}\left(h \eta_{2 x}^{n+\frac{1}{2}}\right) \\
& =\frac{1}{2} \sum_{j=1}^{N} \sum_{k=1}^{N} H 0_{j}\left(c_{k}^{n+1}+c_{k}^{n}\right)\left(\varphi_{j}^{\prime} \varphi_{k}+\varphi_{j} \varphi_{k}^{\prime}\right) .
\end{aligned}
$$

Since

$$
\left(\left(\varphi_{j}^{\prime} \varphi_{k}+\varphi_{j} \varphi_{k}^{\prime}\right), \psi_{i}\right)=C^{(3)}(i, j, k)=C^{(2)}(i, j) \delta_{j k},
$$

terms proportional to $\beta \delta$ can be reduced to single sums like those proportional to $\alpha^{2}, \alpha \beta$ and $\beta^{2}$ discussed in the previous subsections. Finally, one obtains (1) as a system of coupled nonlinear equations $(i=1,2, \ldots, N)$ :

$$
\begin{gathered}
\sum_{j=1}^{N}\left\{\left(a_{j}^{n+1}-a_{j}^{n}\right) C_{i j}^{(1)}+\tau\left[\frac{1}{2}\left(b_{j}^{n+1}+b_{j}^{n}\right) C_{i j}^{(1)}\right.\right. \\
+\left(\alpha \frac{3}{16}\left(a_{j}^{n+1}+a_{j}^{n}\right)^{2}+\beta \frac{1}{12}\left(c_{j}^{n+1}+c_{j}^{n}\right)\right. \\
-\alpha^{2} \frac{1}{64}\left(a_{j}^{n+1}+a_{j}^{n}\right)^{3}+\alpha \beta\left(\frac{13}{192}\left(b_{j}^{n+1}+b_{j}^{n}\right)^{2}\right. \\
\left.+\frac{5}{96}\left(a_{j}^{n+1}+a_{j}^{n}\right)\left(c_{j}^{n+1}+c_{j}^{n}\right)\right)+\beta^{2} \frac{19}{720}\left(e_{j}^{n+1}+e_{j}^{n}\right) \\
-\frac{1}{4} \delta H 0_{j}\left(a_{k}^{n+1}+a_{k}^{n}\right)+\frac{1}{8} \beta \delta H 2_{j}\left(a_{k}^{n+1}+a_{k}^{n}\right) \\
\left.\left.\left.-\frac{1}{8} \beta \delta H 0_{j}\left(c_{j}^{n+1}+c_{j}^{n}\right)\right) C_{i j}^{(2)}\right]\right\}=0, \\
\sum_{j=1}^{N}\left[\left(a_{j}^{n+1}+a_{j}^{n}\right) C_{i j}^{(2)}-\left(b_{j}^{n+1}+b_{j}^{n}\right) C_{i j}^{(1)}\right]=0, \\
\sum_{j=1}^{N}\left[\left(b_{j}^{n+1}+b_{j}^{n}\right) C_{i j}^{(2)}-\left(c_{j}^{n+1}+c_{j}^{n}\right) C_{i j}^{(1)}\right]=0, \\
\sum_{j=1}^{N}\left[\left(c_{j}^{n+1}+c_{j}^{n}\right) C_{i j}^{(2)}-\left(d_{j}^{n+1}+d_{j}^{n}\right) C_{i j}^{(1)}\right]=0,
\end{gathered}
$$

In this case the structures of the vector $X^{n}$ and all matrices remain the same as in 37-39). However, the matrix elements in matrices $A_{a}$ and $A_{c}$ are now different from those in Section 3.2.2, due to the new terms in (44).

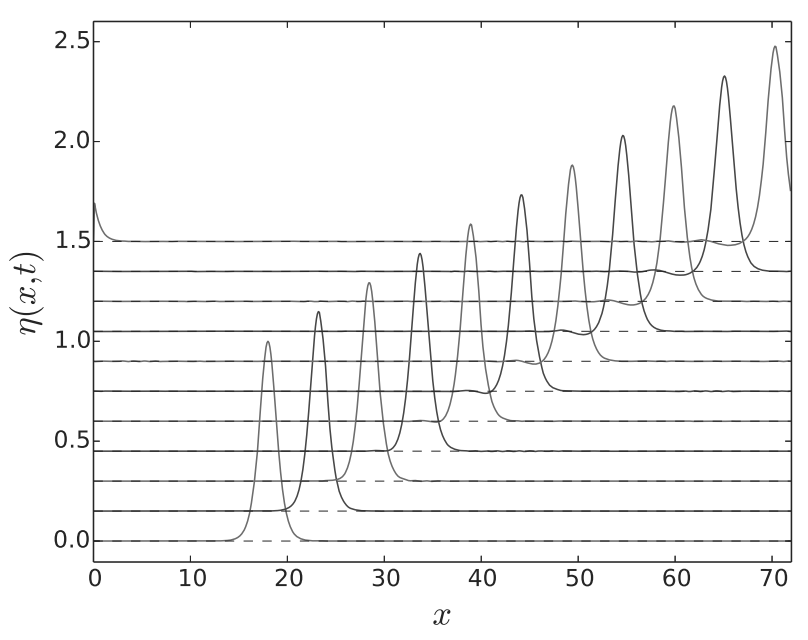

Fig. 1. Time evolution of the initial KdV soliton according to the extended $\mathrm{KdV}$ equation (3). The profiles are obtained by a numerical solution of the set of equations (36). The dashed lines represent the undisturbed fluid surface.

\section{Numerical simulations}

It was demonstrated by Debussche and Printems (1999) that the method described in the previous section works reasonably well for the $\mathrm{KdV}$ equation (7). Our aim was to apply the finite element method in order to numerically solve the second order equations with a flat bottom (3) and with an uneven bottom (11). There exist two kinds of solutions to $\mathrm{KdV}$ equations: soliton (in general, multi-soliton) solutions and periodic solutions, called cnoidal waves (see, e.g. Whitham, 1974; Dingemans, 1997). In Sections 4.1 and 4.2 we present some examples of numerical simulations for soliton solutions, whereas in Section 4.3_some examples for cnoidal solutions.

4.1. Extended KdV equation (3). In Fig. 1 we present several steps of the time evolution of the soliton wave (at $t=0$ it is the $\mathrm{KdV}$ soliton) according the the extended $\mathrm{KdV}$ equation (3) and the numerical scheme (36). The mesh size is $N=720$, with a time step $\tau=\chi^{2}$ and parameters $\alpha=\beta=0.1$. Plotted are the calculated profiles of the wave $\eta\left(x, t_{k}\right)$, where $t_{k}=5 \cdot k$, $k=0,1, \ldots, 10$. In order to avoid overlaps of profiles at different time instants, each subsequent profile is shifted up by 0.15 with respect to the previous one. This convention is used in Figs. 2 and 3 as well. Here and in the next figures the dashed lines represent the undisturbed fluid surface. As the initial condition we chose the standard $\mathrm{KdV}$ soliton centered at $x_{0}=18$. That is, in the applied units,

$$
\eta(x, t=0)=\operatorname{sech}^{2}\left[\frac{\sqrt{3}}{2}\left(x-x_{0}\right)\right] .
$$


Note that since we use scaled variables and the definition (2), the amplitude of the soliton is equal to 1. In Figs. 2,4 we use the same initial conditions.

The soliton motion shown in Fig. 1 is in agreement with the numerical results obtained with the finite difference method by Karczewska et al. (2014b; 2014a). With parameters $\alpha=\beta=0.1$, the resulting distortion of the KdV soliton due to second order terms in (3), (36) is in the form of a small amplitude wavetrain created behind the main wave.

4.2. Uneven bottom. We may ask whether the FEM numerical approach to the extended $\mathrm{KdV}$ (44) is precise enough to reveal the details of soliton distortion caused by a varying bottom. The examples plotted in Figs. 2, 4 show that this is indeed the case. In all the presented calculations the amplitude of the bottom variations is $\delta=$ 0.2 . The bottom profile is plotted as a black line below zero on a different scale than the wave profile.

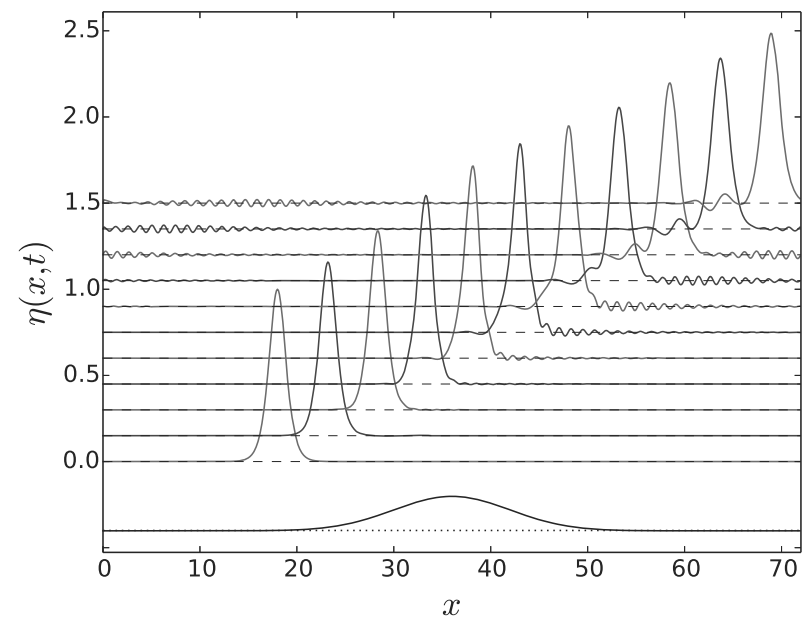

Fig. 2. Time evolution of the initial KdV soliton governed by the extended $\mathrm{KdV}$ equation (1) when the bottom has one hump. Here and in the following figures the dotted line shows the position of (the) undisturbed bottom.

In Fig. 2 the motion of the KdV soliton over a wide bottom hump of a Gaussian shape is presented. Here, the bottom function is

$$
h(x)=\delta \exp \left(-\left(\frac{x-36}{7}\right)^{2}\right) .
$$

In the scaled variables the undisturbed surface of the water (dashed lines) is at $y=0$. The soliton profiles shown in Fig. 2 are almost the same as those obtained with the finite differences method (FDM) used by Karczewska et al. $(2014 b ; 2014 a)$. There are small differences due to a lower precision of our FEM calculations. The FEM allows the use of larger time steps than the FDM. However, in the FEM the computing time grows rapidly with an increase in the number $N$ of the mesh, since calculation of the inverse of the Jacobian $(5 N \times 5 N)$ matrices becomes time consuming.

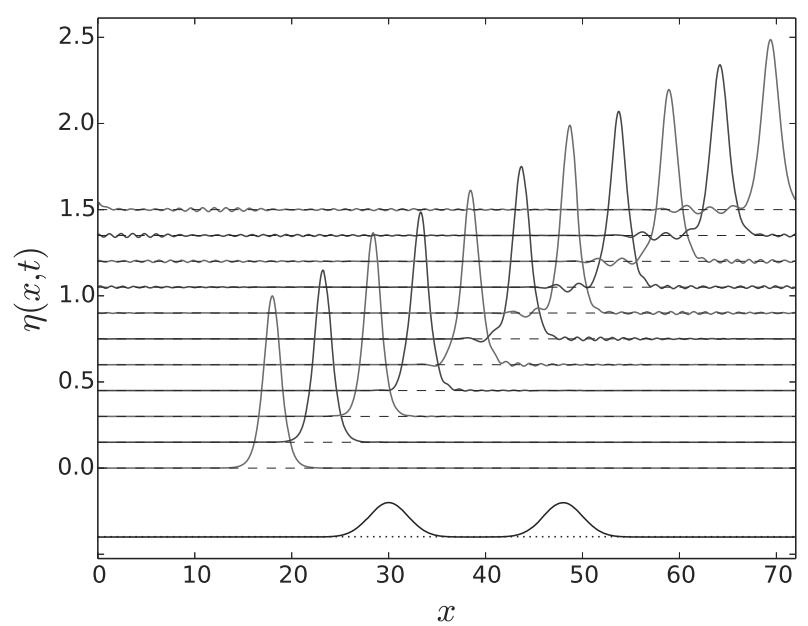

Fig. 3. Time evolution of the initial KdV soliton governed by the extended $\mathrm{KdV}$ equation (1) when the bottom has two narrow humps.

Figure 3 displays the motion of the $\mathrm{KdV}$ soliton above a double humped Gaussian shaped bottom defined by

$h(x)=\delta\left[\exp \left(-\left(\frac{x-30}{6 \sqrt{2}}\right)^{2}\right)+\exp \left(-\left(\frac{x-48}{6 \sqrt{2}}\right)^{2}\right]\right.$.

Here, both the Gaussians are rather narrow and therefore distortions of the wave shape from the ideal soliton are smaller than those in Fig. 2.

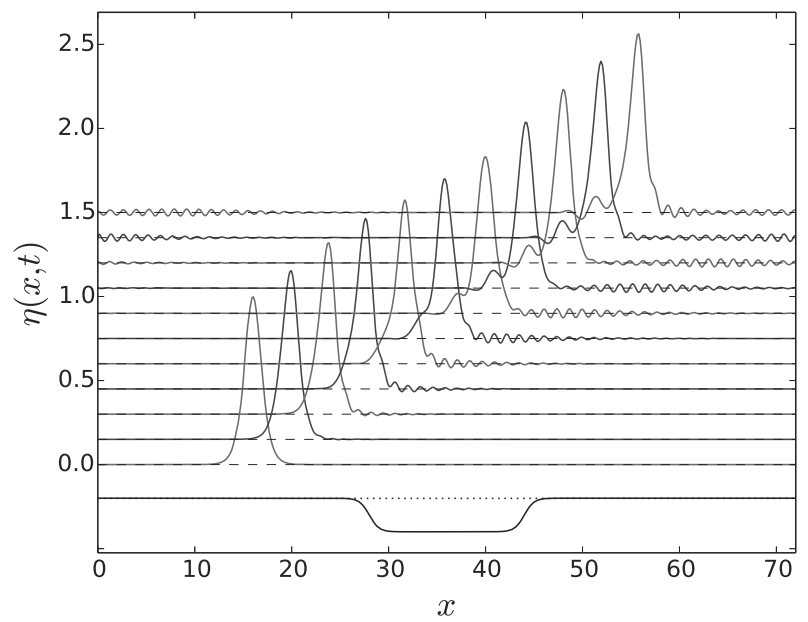

Fig. 4. Time evolution of the initial KdV soliton governed by the extended $\mathrm{KdV}$ equation (1) when the bottom has a well.

In Fig. 4 we see the influence of a bottom well with horizontal size extending the soliton's wavelength. The 
bottom function is chosen as

$$
h(x)=1-\frac{\delta}{2}[\tanh (x-28)+\tanh (44-x)]
$$

symmetric with respect to the center of the $x$ interval. Figure 4 shows that during the motion above smooth obstacles two effects appear. First, some additional 'waves' of small amplitude, but moving faster than the main solitary wave, appear. Second, a wave of smaller amplitude and smaller velocity appears behind the main wave. Both these properties were observed and described in detail in our previous paper (Karczewska et al., 2014a).

4.3. Motion of cnoidal waves. Cnoidal solutions to the KdV equation are expressed by the Jacobi elliptic $\mathrm{cn}^{2}$ function. The explicit formula for cnoidal solutions (see, e.g., Dingemans, 1997) is

$$
\eta(x, t)=\eta_{2}+H \mathrm{cn}^{2}\left(\frac{x-c t}{\Delta} \mid m\right),
$$

where

$$
\eta_{2}=\frac{H}{m}\left(1-m-\frac{E(m)}{K(m)}\right), \quad \Delta=h \sqrt{\frac{4 m h}{3 H}},
$$

and

$$
c=\sqrt{g h}\left[1+\frac{H}{m h}\left(1-\frac{m}{2}-\frac{3 E(m)}{2 K(m)}\right)\right] .
$$

The solution (45)-47) is written in dimensional quantities, where $H$ is the wave height, $h$ is the mean water depth, $g$ is the gravitational acceleration and $m$ is an elliptic parameter. $K(m)$ and $E(m)$ are complete elliptic integrals of the first and second kinds, respectively. The value of $m \in[0,1]$ governs the shape of the wave.

As $m \rightarrow 0$, the cnoidal solution converges to a cosine function. As $m \rightarrow 1$, the cnoidal wave forms peaked crests and flat troughs, such that for $m=1$ the distance between crests increases to infinity and the cnoidal wave converges to a soliton solution.

For (1) and (3) we have to express the formulas 45-47) in dimensionless variables.

Figure 5 shows the time evolution of the cnoidal wave according to the extended $\mathrm{KdV}$ equation (3), that is, the second order $\mathrm{KdV}$ equation with a flat bottom. The parameters of the simulation are $\alpha=\beta=0.14, m=$ $1-10^{-16}$. With this value of $m$ the wavelength of the cnoidal wave is equal to $d \approx 75.1552$ dimensionless units, and calculations were performed on the interval of that length, $x \in[0,75.1552]$, with a periodic boundary condition. The mesh size was taken as $N=752$. The initial position of the wave peak was chosen at the center of the given interval, that is, $x_{0}=37.5776$. The explicit form of the initial condition in this case was $\eta(x, t=$

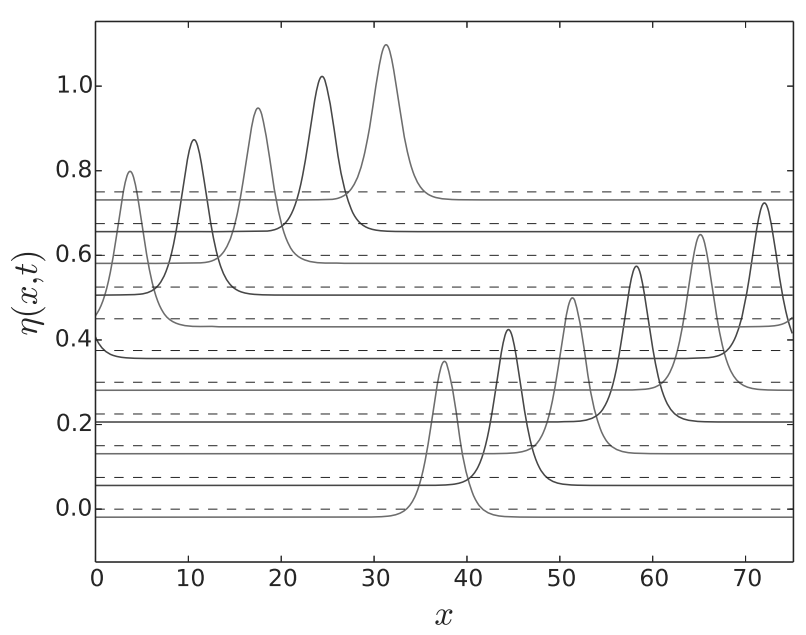

Fig. 5. Time evolution of the initial $\mathrm{KdV}$ cnoidal wave governed by the extended $\mathrm{KdV}$ equation (3) and the numerical scheme 36 .

$0)=-0.0189862+0.368486 \mathrm{cn}^{2}\left(\frac{x-x_{0}}{1.90221} \mid m\right)$. Profiles of the wave are plotted at time instants $t_{k}=10 \cdot k$, where $k=0,1, \ldots, 8$. Since the amplitudes of cnoidal waves are less than 1 , the vertical shift for the sequential profiles in Figs. 5, 8 is chosen to be 0.075 .

In Fig. 6 we display the initially cnoidal wave moving over an extended, almost flat hump. In this simulation the value of parameters $\alpha, \beta, m$ and interval $x$ are the same as in the previous figure. Since we consider here the motion over an uneven bottom defined by the function

$$
\begin{aligned}
h(x)=\frac{1}{2}[-\tanh ( & \left.2(x-8.6)-\frac{1}{2}\right) \\
& \left.+\tanh \left(2(x-66.5552)-\frac{1}{2}\right)\right],
\end{aligned}
$$

the evolution was calculated according to Eqn. (1) and the numerical scheme (44). Profiles of the wave are plotted at time instants $t_{k}=10 \cdot k$, where $k=0,1, \ldots, 8$. Figure 6 shows that during the wave motion over the obstacle a kind of slower wave with smaller amplitude is created following the main peak.

In Fig. 8 we present the initially cnoidal wave moving over an extended, almost flat hump. In this simulation, $m=1-10^{-8}$. The initial condition is given by

$$
\eta(x, t=0)=0.0359497+0.368486 \mathrm{cn}^{2}\left(\frac{x-x_{0}}{1.90221} \mid m\right)
$$

with $x_{0}=20.1571$. Because $m$ is smaller than in the previous cases, the wavelength $d$ of the cnoidal wave is also smaller, $d \approx 40.3241$. Calculations were made on the interval $x \in[0,2 d]$ with $N=807$. Profiles of the wave are plotted at time instants $t_{k}=10 \cdot k$, where $k=$ $0,1, \ldots, 8$. Figure 8 shows qualitatively similar features to those in Fig. 6 . 


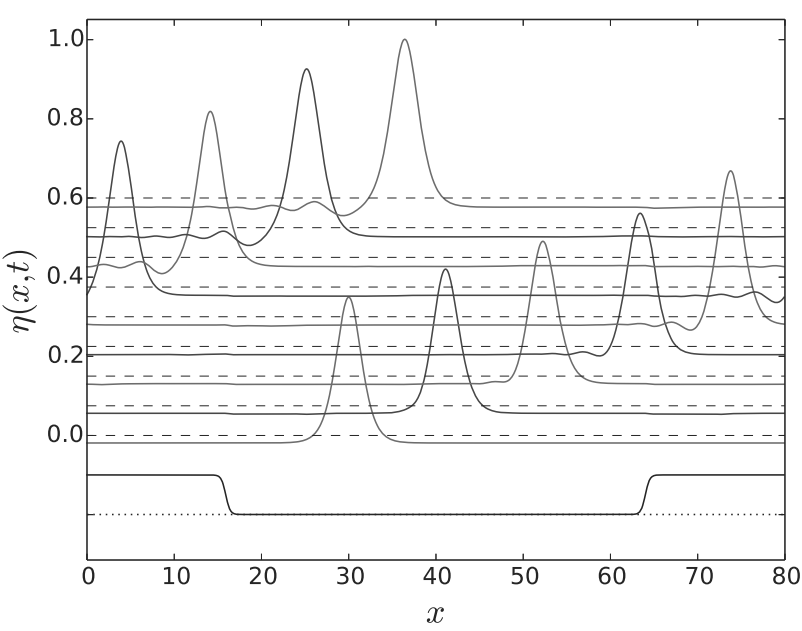

Fig. 6. Time evolution of the initial $\mathrm{KdV}$ cnoidal wave governed by the extended $\mathrm{KdV}$ equation (1). The bottom function is here $h(x)=\frac{1}{2}\left[-\tanh \left(2(x-8.6)-\frac{1}{2}\right)+\tanh (2(x-\right.$ $\left.\left.66.5552)-\frac{1}{2}\right)\right]$.

4.4. Precision of numerical calculations. The KdV equation (6) or (7) is unique since it possesses an infinite number of invariants (see, e.g., Miura et al., 1968; Drazin and Johnson, 1989). The lowest invariant,

$$
I_{1}=\int_{-\infty}^{+\infty} \eta \mathrm{d} x,
$$

represents the conservation law for the mass (volume) of the liquid. The second,

$$
I_{2}=\int_{-\infty}^{+\infty} \eta^{2} \mathrm{~d} x
$$

is related to momentum conservation, and the third,

$$
I_{3}=\int_{-\infty}^{+\infty}\left(\eta^{3}-\frac{1}{3} \eta_{x}^{2}\right) \mathrm{d} x
$$

is related to energy conservation. However, as pointed by Ablowitz and Segur (1979), Ali and Kalisch (2014) or Karczewska et al. (2015), the relations between $I_{2}$ and momentum and $I_{3}$ and energy are more complex.

Approximate conservation of these invariants often serves as a test of the precision of numerical simulations. However, this is not the case for the second order $\mathrm{KdV}$ type equations (1) and (3). It was noted by Karczewska et al. (2015) that $I_{1}$ is an invariant of Eqns. (1) and (3), but $I_{2}$ and $I_{3}$ are not invariants. Therefore, only $I_{1}$ can be used as a test for the precision of numerical calculations of waves moving according to second order extended KdV equations. In all the presented calculations the precision of the numerical values of $I_{1}$ was consistently high, i.e.,

$$
\frac{I_{1}(t)-I_{1}(0)}{I_{1}(0)} \leq 10^{-6}
$$

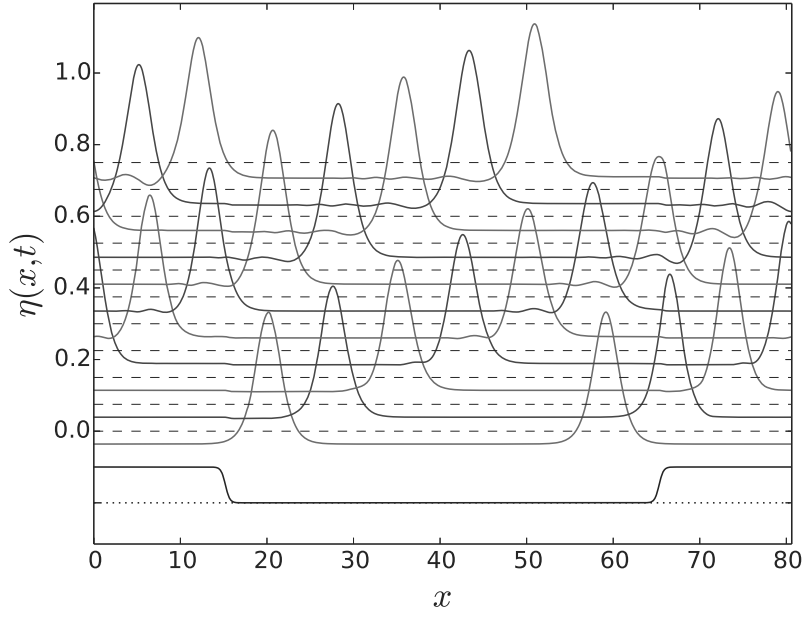

Fig. 7. Time evolution of the initial $\mathrm{KdV}$ cnoidal wave governed by the extended $\mathrm{KdV}$ equation (1). The bottom function is here $h(x)=\frac{1}{2}\left[-\tanh \left(2(x-13.3)-\frac{1}{2}\right)+\tanh (2(x-\right.$ 67) $\left.-\frac{1}{2}\right)$ ]

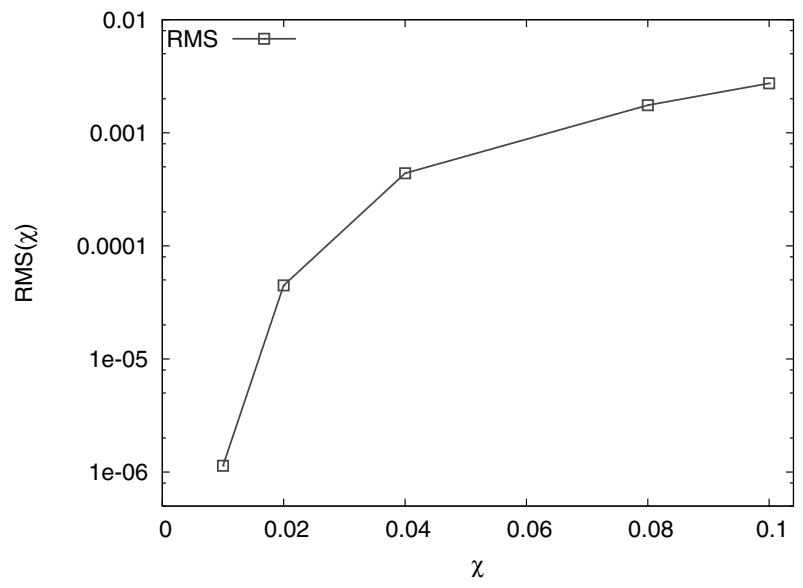

Fig. 8. Precision of numerical calculations for the KdV equation in the fixed frame as a function of mesh size.

Wave motion according to $\mathrm{KdV}$ and extended (second order) $\mathrm{KdV}$ equations is usually calculated in the reference frame moving with the natural velocity $c=1$ in scaled dimensionless variables (in the original variables $c=\sqrt{g h}$ ). The $\mathrm{KdV}$ and extended $\mathrm{KdV}$ equations for a moving reference frame are obtained by the transformation $\hat{x}=(x-t), \hat{t}=t$, which removes the term $\eta_{x}$ from the Eqn. (3). Then the soliton velocity in the fixed frame is proportional to $1+\alpha / 2$, whereas in the moving frame it is proportional to $\alpha / 2$. Therefore, for the value of $\alpha=0.1$ the distance covered by a soliton in the moving frame is $(\alpha / 2) /(1+\alpha / 2)=1 / 21$ times shorter than the distance covered in the fixed frame for the same duration. Then, with the same number of the mesh points $N$ the mesh size $\chi$ can be more than 20 times smaller assuring much higher precision of calculation in 
the moving frame at the same operational cost. For instance, Debussche and Printems (1999) obtained good precision for motion of a $\mathrm{KdV}$ soliton with the FEM method using $N=200, \chi=0.01$ and time step $\tau=\chi$ on the interval $x \in[0,2]$.

The precision of the FEM method in the fixed frame can be tested by calculation of a root mean square (RMS) of deviations of wave profile obtained numerically from those generated by the analytic solution. Denote by $\eta_{i}^{\text {anal }}(t)$ and $\eta_{i}^{\text {num }}(t)$ the values of the solutions at given mesh point $i$ and time instant $t$, analytic and numerical, respectively. Then the RMS is expressed as

$$
\operatorname{RMS}(\chi, t)=\left(\frac{1}{N} \sum_{i=1}^{N}\left(\eta_{i}^{\text {anal }}(t)-\eta_{i}^{\text {num }}(t)\right)^{2}\right)^{1 / 2}
$$

We checked our implementation of the FEM on the interval $x \in[0,20]$ using several different sizes $\chi$ of the mesh and several time values. Figure 8 displays the RMS (48) values for $t=10$. It shows that deviations from the analytic solution decrease substantially with decreasing $\chi$. Small $\chi$ assures very high precision in numerical simulations, although at the expense of large computation time. Another tests (not shown here) in which $\chi$ was fixed and the RMS was calculated as a function of time showed that for $\tau=\chi^{2}$ the RMS increases with time linearly and very slowly.

When the bottom is not flat, simulations have to be done in the fixed reference frame. For our purposes we needed to choose the $x$ intervals of the order of 70 or 80. Even for $\chi=0.1$ the size of the Jacobian matrices 39 reaches $(4000 \times 4000)$ and its inversion is time consuming. In a compromise between numerical precision and reasonable computing times we made our simulations with $\chi=0.1$. This choice resulted in about one week of computing time for a single run on a cluster. In spite of the insufficient precision the results presented in Figs. 1-7 reproduce details of evolution known from our previous studies, obtained with the finite difference method. These details, resulting from second order terms in the extended $\mathrm{KdV}$ (3), are seen in Fig. 1 as a wavetrain of small amplitude created behind the main one (compare it with Fig. 2 of Karczewska et al. (2014a)). A similar wavetrain behind the main one was observed in numerical simulations by Marchant and Smyth (1996); see, e.g., Fig. 2 therein. For waves moving with the presence of a bottom obstacle these secondary waves behind the main one are amplified by interaction with the bottom and new faster secondary waves appear (see, e.g., Figs. 2-4). These effects were already observed by us (Karczewska et al., 2014a, Figs. 6 and 7).

\section{Conclusions}

The main conclusions of our study can be summarized as follows.

- A weak formulation of the finite element method (FEM) for the extended KdV equation (3) can be effectively used for numerical calculations of the time evolution of both soliton and cnoidal waves when calculations are done in a moving frame.

- Since numerical calculations for Eqn. (1) have to be performed in a fixed frame, the presented FEM method is not as effective as the FDM method used by us in our previous papers because the computer time necessary for obtaining sufficiently high precision becomes impractical. On the other hand, the presented results (though not as precise as FDM ones) exhibit all secondary structures generated by higher order terms of the equations.

- First tests of numerical solutions to second order $\mathrm{KdV}$ type equations with a stochastic term seem to be very promising (Karczewka et al., 2016).

\section{Acknowledgment}

The authors would like to thank anonymous referees for several helpful suggestions and remarks that affected the article content.

\section{References}

Ablowitz, M. and Segur, H. (1979). On the evolution of packets of water waves, Journal of Fluid Mechanics 92(4): 691-715.

Ali, A. and Kalisch, H. (2014). On the formulation of mass, momentum and energy conservation in the $\mathrm{KdV}$ equation, Acta Applicandae Mathematicae 133(1): 113-131.

Bona, J., Chen, H., Karakashian, O. and Xing, Y. (2013). Conservative, discontinuous-Galerkin methods for the generalized Korteweg-de Vries equation, Mathematics of Computation 82(283): 1401-1432.

Burde, G. and Sergyeyev, A. (2013). Ordering of two small parameters in the shallow water wave problem, Journal of Physics A 46(7): 075501.

Cui, Y. and Ma, D. (2007). Numerical method satisfying the first two conservation laws for the Korteweg-de Vries equation, Journal of Computational Physics 227(1): 376-399.

Debussche, A. and Printems, I. (1999). Numerical simulation of the stochastic Korteweg-de Vries equation, Physica $D$ 134(2): 200-226.

Dingemans, M. (1997). Water Wave Propagation over Uneven Bottoms, World Scientific, Singapore.

Drazin, P.G. and Johnson, R.S. (1989). Solitons: An Introduction, Cambridge University Press, Cambridge. 
Fornberg, B. and Whitham, G.B. (1978). A numerical and theoretical study of certain nonlinear wave phenomena, Philosophical Transactions A of the Royal Society 289(1361): 373-404.

Goda, K. (1975). On instability of some finite difference schemes for the Korteweg-de Vries equation, Journal of the Physical Society of Japan 39(1): 229-236.

Green, A.E. and Naghdi, P.M. (1976). A derivation of equations for wave propagation in water of variable depth, Journal of Fluid Mechanics 78(2): 237-246.

Grimshaw, R. (1970). The solitary wave in water of variable depth, Journal of Fluid Mechanics 42(3): 639-656.

Grimshaw, R.H.J. and Smyth, N.F. (1986). Resonant flow of a stratified fluid over topography, Journal of Fluid Mechanics 169: 429-464.

Grimshaw, R., Pelinovsky, E. and Talipova, T. (2008). Fission of a weakly nonlinear interfacial solitary wave at a step, Geophysical and Astrophysical Fluid Dynamics 102(2): 179-194.

Infeld, E. and Rowlands, G. (2000). Nonlinear Waves, Solitons and Chaos, 2nd Edition, Cambridge University Press, Cambridge.

Kamchatnov, A.M., Kuo, Y.H., Lin, T.C., Horng, T.L., Gou, S.C., Clift, R., El, G.A. and Grimshaw, R.H.J. (2012). Undular bore theory for the Gardner equation, Physical Review E 86: 036605 .

Karczewska, A., Rozmej, P. and Infeld, E. (2014a). Shallow-water soliton dynamics beyond the Korteweg-de Vries equation, Physical Review E 90: 012907.

Karczewska, A., Rozmej, P. and Rutkowski, L. (2014b). A new nonlinear equation in the shallow water wave problem, Physica Scripta 89(5): 054026.

Karczewska, A., Rozmej, P. and Infeld, E. (2015). Energy invariant for shallow water waves and the Korteweg-de Vries equation: Doubts about the invariance of energy, Physical Review E 92: 053202.

Karczewska, A., Szczeciński, M., Rozmej, P., and Boguniewicz, B. (2016). Finite element method for stochastic extended KdV equations, Computational Methods in Science and Technology 22(1): 19-29.

Kim, J.W., Bai, K.J., Ertekin, R.C. and Webster, W.C. (2001). A derivation of the Green-Naghdi equations for irrotational flows, Journal of Engineering Mathematics 40: 17-42.

Marchant, T. and Smyth, N. (1990). The extended Korteweg-de Vries equation and the resonant flow of a fluid over topography, Journal of Fluid Mechanics 221(1): 263-288.

Marchant, T. and Smyth, N. (1996). Soliton interaction for the extended Korteweg-de Vries equation, IMA Journal of Applied Mathematics 56: 157-176.

Mei, C. and Le Méhauté, B. (1966). Note on the equations of long waves over an uneven bottom, Journal of Geophysical Research 71(2): 393-400.

Miura, R.M., Gardner, C.S. and Kruskal, M.D. (1968). Korteweg-de Vries equation and generalizations, II: Existence of conservation laws and constants of motion, Journal of Mathematical Physics 9(8): 1204-1209.
Nadiga, B., Margolin, L. and Smolarkiewicz, P. (1996) Different approximations of shallow fluid flow over an obstacle, Physics of Fluids 8(8): 2066-2077.

Nakoulima, O., Zahibo, N. Pelinovsky, E., Talipova, T. and Kurkin, A. (2005). Solitary wave dynamics in shallow water over periodic topography, Chaos 15(3): 037107.

Pelinovsky, E., Choi, B., Talipova, T., Woo, S. and Kim, D. (2010). Solitary wave transformation on the underwater step: Theory and numerical experiments, Applied Mathematics and Computation 217(4): 1704-1718.

Pudjaprasetya, S.R. and van Greoesen, E. (1996) Uni-directional waves over slowly varying bottom, II: Quasi-homogeneous approximation of distorting waves, Wave Motion 23(1): 23-38.

Remoissenet, M. (1999). Waves Called Solitons: Concepts and Experiments, Springer, Berlin.

Skogstad, J. and Kalisch, H. (2009). A boundary value problem for the $\mathrm{KdV}$ equation: Comparison of finite difference and Chebyshev methods, Mathematics and Computers in Simulation 80(1): 151-163.

Smyth, N.F. (1987). Modulation theory solution for resonant flow over topography, Proceedings of the Royal Society of London A 409(1836): 79-97.

Taha, T.R. and Ablowitz, M.J. (1984). Analytical and numerical aspects of certain nonlinear evolution equations III: Numerical, Korteweg-de Vries equation, Journal of Computational Physics 55(2): 231-253.

van Greoesen, E. and Pudjaprasetya, S.R. (1993). Uni-directional waves over slowly varying bottom, I: Derivation of a KdV-type of equation, Wave Motion 18(4): 345-370.

Whitham, G.B. (1974). Linear and Nonlinear Waves, Wiley, New York, NY.

Yi, N., Huang, Y. and Liu, H. (2013). A direct discontinous Galerkin method for the generalized Korteweg-de Vries equation: Energy conservation and boundary effect, Journal of Computational Physics 242: 351-366.

Yuan, J.-M., Shen, J. and Wu, J. (2008). A dual Petrov-Galerkin method for the Kawahara-type equations, Journal of Scientific Computing 34: 48-63.

Zabusky, N.J. and Kruskal, M.D. (1965). Interaction of 'solitons' in a collisionless plasma and the recurrence of initial states, Physical Review Letters 15(6): 240-243.

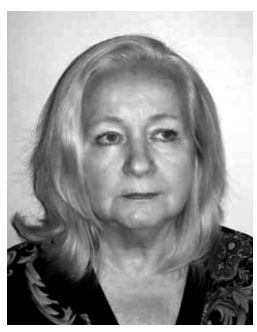

Anna Karczewska is a professor at the Faculty of Mathematics, Computer Science and Econometrics, University of Zielona Góra. She holds an M.Sc. degree from the Warsaw University of Technology, a Ph.D. degree from Jagiellonian University, Cracow, and a D.Sc. degree from the University of Zielona Góra. Her fields of interest include deterministic and stochastic integral equations, in particular Volterra equations of integral and fractional order, fluid dynamics and Korteweg-de Vries type equations. 


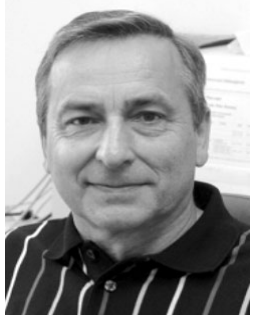

Piotr Rozmej is a full professor at the Faculty of Physics and Astronomy, University of Zielona Góra, the head of the Division of Mathematical Methods in Physics. He holds an M.Sc. degree from Maria Curie-Skłodowska University (UMCS), Lublin, a Ph.D. degree from Warsaw University, and a D.Sc. degree from UMCS. He became a professor in 1992 and a full professor in 1997. He has spent several years working as a senior researcher at the Joint Institute for $\mathrm{Nu}-$ clear Research (Dubna, USSR), Gesellschaft für Schwerionenforschung (Darmstadt, Germany) and Institut des Sciences Nuclaires (Grenoble, France). He has worked in several fields of theoretical nuclear physics like nuclear structure, nuclear reactions, properties of heavy and superheavy nuclei. Other areas of his scientific activity include quantum optics, in particular wave packet dynamics, deterministic chaos, nonlinear dynamics, solitons, numerical modeling and simulations.

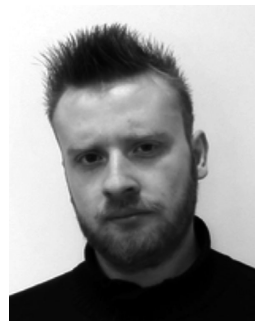

Maciej Szczeciński holds an M.Sc. degree in economics from the Wrocław University of Economics and an M.Sc. degree in mathematics obtained at the Faculty of Mathematics, Computer Science and Econometrics, University of Zielona Góra. At present he is Ph.D student at this faculty and is working on his thesis under Professor Anna Karczewska's supervision.

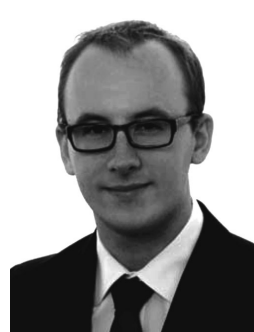

Bartosz Boguniewicz, Faculty of Physics and Astronomy, University of Zielona Góra. He finished his M.Sc. thesis on Korteweg-de Vries type equations under the supervision of Professor Piotr Rozmej in July 2016.

Received: 2 June 2015

Revised: 20 December 2015 Accepted: 8 March 2016 